\title{
Forecasting Taiwan's GDP by the Novel Weighted Average Nonlinear Grey Bernoulli Model
}

\author{
Pei-Han Hsin
}

\begin{abstract}
In grey forecasting model, the average relative percentage error is an important criterion for assessing the forecasting precision. However, it seldom considers in-sample and out-of-sample tests at the same time. Besides, overestimation or underestimation usually exists when the original data has a rising or falling trend. Thus, this study proposes weighted average nonlinear grey Bernoulli model to solve above two problems. A weighted average moderates the effect of overestimation or underestimation. A new criterion which considers in-sample and out-of-sample tests at the same time indicates that the weighted average nonlinear grey Bernoulli model has the smallest modeling error. Finally, the proposed method is used to forecast Taiwan's GDP. The results show that Taiwan's GDP is steadily growing. It may serve as valuable information for policy makers and investors.
\end{abstract}

Index Terms - Grey forecasting, nonlinear grey bernoulli model, GDP, weighted average.

\section{INTRODUCTION}

Grey forecasting [1] has been developing for more than 20 years and its evolution never stops. Deng [2] proposes grey forecasting which only needs four data and is suitable for short-term forecasts. Grey forecasting has been successfully applied in many fields, including finance [3], agriculture [4], transportation [5], electric power load [6], semiconductor industry [7], economy [8] and so on.

The traditional grey forecasting is sometimes with its unsatisfied prediction precision. Thus, the researchers develop the hybrid grey model for improving the forecasting ability, for example, Grey-Markov model [9], Grey-Fuzzy model [10], Grey-Taguchi model [11] and so on. Besides, some researchers try to change the stucture of grey forecasting models, including Grey Verhulst model [12], the nonlinear grey Bernoulli model (NGBM) [13-14], and Nash NGBM [15]. On the other hands, some researchers use particle swarm optimization algorithm [16-17] or genetic algorithm [18] to optimize grey model .

In NGBM [13], [14], the coefficient $\mathrm{p}$ is always set as 0.5 . In fact, the coefficient $\mathrm{p}$ may be between 0 and 1 . Besides, overestimation or underestimation are common in forecasting practics. For this reason, this study tries to find out all forecast values as $\mathrm{p}$ changes and then adopts arithmetic average to moderate the effect of overestimation

Manuscript received October 10, 2015; revised December 10, 2015.

Pei-Han Hsin is with the Department of International Business, Cheng Shiu University, Kaohsiung City, Taiwan (R.O.C.) (e-mail: phhsin@gmail.com). and underestimation.

This study also develops a new criterion which considers in-sample error and out-of-sample tests. Because those two types of error sometimes are trade-off, it is not enough to judge a forecasting model only by in-sample error, such as in Chen's articles [14], [15].

This paper is organized as follows. Section 2 introduces the mathematics of weighted average NGBM, and defines the forecasting relative percentage error. Section 3 forecasts Taiwan's GDP by the proposed method. Finally, section 4 presents conclusion.

\section{MATHEMATICAL METHOdOLOGY}

The procedures for obtaining $\operatorname{NGBM}(1,1)$ are elaborated below:

Step 1: Assume that the original series of data with $m$ entries is:

$$
X^{(0)}(1, m)=\left\{x^{(0)}(k) \mid x^{(0)}(k) \geq 0, k=1,2, \ldots, m\right\}
$$

where raw matrix $X^{(0)}$ represents the non-negative original time series data.

Step 2: Construct $X^{(1)}(1, m)$ by one time accumulated generation operation (1-AGO). Thus, $X^{(1)}(1, m)$ is

$$
X^{(1)}(1, m)=\left\{x^{(1)}(k)=\sum_{i=1}^{k} x^{(0)}(k), \mid x^{(1)}(k) \geq 0, k=1,2, \ldots, m\right\}
$$

Step 3: The grey differential equation of $\operatorname{NGBM}(1,1)$ has following form [13]-[15].

$$
\frac{d \hat{x}^{(1)}}{d t}+\alpha \hat{x}^{(1)}=\beta\left[\hat{x}^{(1)}\right]^{n},
$$

where $n$ is any real number but 1 .

The background value is $\hat{x}^{(1)}(t) \cong p x^{(1)}(k)+(1-p) x^{(1)}(k+1)=z^{(1)}(k)$ and $p \in[0,1]$.

Step 4: A discrete form of (3) is described as

$$
x^{(0)}(k)+\alpha z^{(1)}(k)=\beta\left[z^{(1)}(k)\right]^{n}, k=2,3,4, \ldots
$$

By the least square method, the parameters $\alpha$ and $\beta$ become

$$
\left\lfloor\begin{array}{l}
\alpha \\
\beta
\end{array}\right\rfloor=\left(Z^{T} Z\right)^{-1} Z^{T} X,
$$

where $Z$ and $X$ are defined as follows. 


$$
Z=\left[\begin{array}{cc}
-z^{(1)}(2) & {\left[z^{(1)}(2)\right]^{n}} \\
-z^{(1)}(3) & {\left[z^{(1)}(3)\right]^{n}} \\
\vdots & \vdots \\
-z^{(1)}(m) & {\left[z^{(1)}(m)\right]^{n}}
\end{array} \mid, X=\left[\begin{array}{c}
x^{(0)}(2) \\
x^{(0)}(3) \\
\vdots \\
x^{(0)}(m)
\end{array} \mid\right.\right.
$$

Step 5: The corresponding particular solution of (4) is

$$
\hat{x}^{(1)}(k+1)=\left[\left(x^{(0)}(1)^{1-n}-\frac{\beta}{\alpha}\right) e^{-\alpha k}+\frac{\beta}{\alpha}\right]^{1 /(1-n)}, k=1,2,3, \ldots
$$

Step 6: Calculate $\hat{x}^{(0)}(k+1)$ which is defined as

$$
\hat{x}^{(0)}(k+1)=\hat{x}^{(1)}(k+1)-\hat{x}^{(1)}(k)
$$

Step 7: In the gray model, the main criterion for assessing forecasting accuracy is relative percentage error which compare the fitted and actual values. The relative percentage error (RPE) is defined as

$$
R P E=\xi(k)=\frac{x^{(0)}(k)-\hat{x}^{(0)}(k)}{x^{(0)}(k)} \times 100 \%
$$

and the average relative percentage error (ARPE) is

$$
A R P E=\zeta(k)=\frac{1}{m-1} \sum_{k=2}^{m}|\xi(k)|
$$

Generally speaking, the gery forecasting modes with the smallest ARPE is regarded as the best forecasting model.

Step 8: Consider in-sample and out-of-sample tests.

This study uses in-sample and out-of-sample tests to assess the performance of a full model.

Suppose that the ARPE of in-sample test is $\zeta_{\text {in }}$, while the ARPE of out-of-sample test is $\zeta_{\text {out }}$. The full model error $\left(\zeta_{\text {model }}\right)$ is defined as

$$
\zeta_{\bmod e l}=\operatorname{Max}\left\{\zeta_{\text {in }}, \zeta_{\text {out }}\right\}
$$

Those grey forecasting models which have the smallest in-sample error don't guarantee to have the smallest in-sample error. Sometimes, there is trade-off relationship between $\zeta_{\text {in }}$ and $\zeta_{\text {out }}$. Therefore, the criterion of (11) become useful to evaluate the forecast performance. The forecasting model which has the smallest $\zeta_{\text {model }}$ is called the best forecasting model.

Step 9: Calculate the optimal $n$.

Consider the following optimization problem.

$$
\operatorname{Min} \zeta\left(n \mid p, X^{(0)}(1, m)\right)
$$

$p \in[0,1], n \in R$. The original series is $X^{(0)}(1, m)$. The solution of above optimization problem is

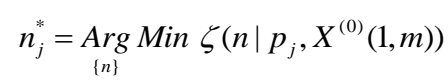

where $p_{j} \in[0,1]$.

Step 10: Seek for all $n_{j}^{*}$ for a given $p_{j}$.
Set $p \in\left\{p_{1} \ldots p_{j} \ldots p_{J}\right\}, j=1,2, \ldots, J$. For a given $p_{j}$, figure out the forecast value $\hat{x}_{p_{j}}(k+1)$.

Step 11: Calculate the weighted average for the forecast value $x(k+1)$ to moderate the effect overestimation and underestimation. Thus,

$$
\tilde{x}^{(0)}(k+1)=\left\{\sum_{j=1}^{j=J} \hat{x}_{p_{j}}^{(0)}(k+1)\right\} / J
$$

\section{FORECASTING TAIWAN'S GDP}

Forecasting Twain's GDP is selected as a case study. GDP annual data is obtained from the website of the Ministry of Economic Affairs of Taiwan. The data period is from 2009 to 2014. The unit of GDP is million US dollars. There are two stages to finish forecasting GDP.

In the first stage, the author develops candidate models and uses in-sample and out-of-sample tests to assess models. To develop candidate models, $\operatorname{NGBM}(1,1)$ appears with different $p$. This study assumes that $p$ can be $0.1,0.3,0.5,0.7$ and 0.9. Besides, this study proposes weighted average $\operatorname{NGBM}(1,1)$ as a new candidate model.

The data from 2009 to 2013 are used to construct $\operatorname{NGBM}(1,1)$ with in-sample error. The data in 2014 acts as out-of-sample test. All empirical results are showed in Table I. The fitted value is overestimation as $\xi(k) \%$ is negative. Underestimation exists as $\xi(k) \%$ is positive. For example, as p equals to 0.7 , the forecast values are $453905.74,487781.13$, $503250.38,508853.24$ and 508340.42 from 2010 to 2014 , respectively. The signs of $\xi(k) \%$ are,,,---+ and + . It means that the forecast values are with overestimation in 2010, 2011 and 2012, and with underestimation in 2013 and 2014. As p equals to 0.5 , overestimation exist only in 2012 . Thus, the method of weighted average balances the effect of underestimation and overestimation. Using (11) as a criterion to assess grey forecasting models, we find that ARPE of the six candidate modes are $1.8583 \%, 1.1464 \%, 3.1960 \%$, $4.0119 \%, 3.5868 \%$ and $1.0407 \%$. The weighted average $\operatorname{NGBM}(1,1)$ has the smallest model error.

Moreover, Chen's NGBM [14-15] has the smallest in-sample error $(0.6163 \%)$ as $\mathrm{p}$ is 0.5 , but out-of-sample error is not the smallest $(3.1960 \%)$. It demonstrates that those grey forecasting models with the smallest in-sample error don't guarantee to have the smallest in-sample error.

The results indicate that weighted average $\operatorname{NGBM}(1,1)$ is the most suitable for acting as a forecasting model.

In the second stage, we use GDP data from 2010 to 2014 to predict GDP from 2015 to 2017 . To obtain the forecast values of weighted average $\operatorname{NGBM}(1,1)$, it is necessary to calculate the forecast values of $\operatorname{NGBM}(1,1)$ with different $p$. All results show in Table II. It's worth noting that ARPE of the proposed model is $0.1699 \%$ which is less than any other $\operatorname{NGBM}(1,1)$.

The predicted values of Taiwan's GDP are 547271.91, 567295.77 and 588638.33 from 2015 to 2017, respectively. The evidences show that Taiwan's GDP is steadily growing. 
TABLE I: ASSESSING THE FORECAST EFFICIENCY OF WEIGHTED AVERAGE NGBM(1,1) USING IN-SAMPLE AND OUT-OF-EXAMPLE TESTS

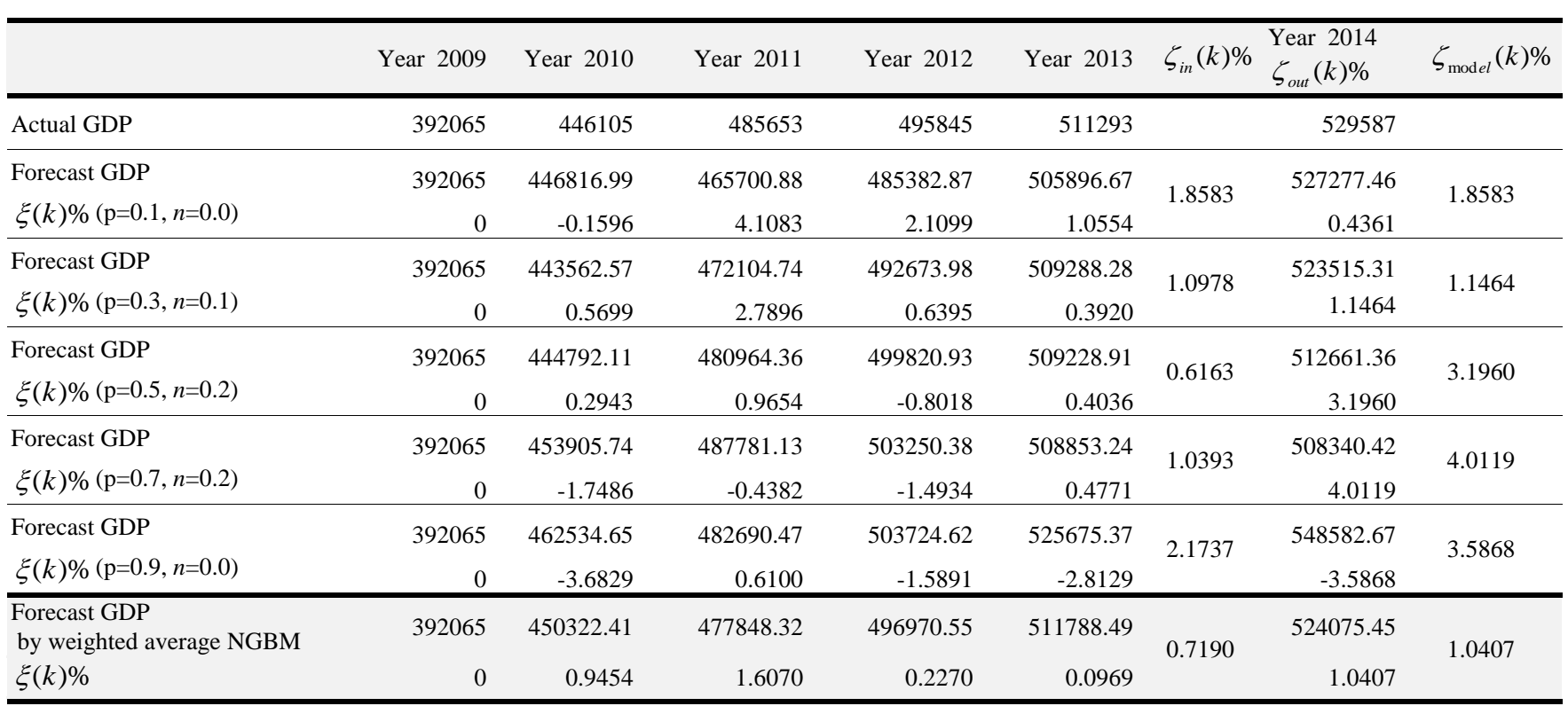

TABLE II: The ForeCAST VALUE of TAIWAN's GDP USING THE WeIGHTED AVERAGE MGBM $(1,1)$

\begin{tabular}{lrrrrrrrrr}
\hline Year & 2010 & 2011 & 2012 & 2013 & 2014 & 2015 & 2016 & 2017 & $\zeta(k) \%$ \\
\hline Actual GDP & 446105 & 485653 & 495845 & 511293 & 529587 & & & & \\
\hline Forecast GDP & 446105 & 487567.30 & 490823.53 & 503767.90 & 521569.99 & 542700.32 & 566517.95 & 592725.42 & 1.0981 \\
$\xi(k) \%(\mathrm{p}=0.1, \mathrm{n}=-0.1)$ & 0 & -0.3942 & 1.0127 & 1.4718 & 1.5138 & & & & \\
\hline Forecast GDP & 446105 & 480770.30 & 494939.05 & 509525.36 & 524541.55 & 540000.27 & 555914.58 & 572297.90 & 0.6216 \\
$\xi(k) \%(\mathrm{p}=0.3, \mathrm{n}=0.0)$ & 0 & 1.0054 & 0.1827 & 0.3457 & 0.9527 & & & \\
\hline $\begin{array}{l}\text { Forecast GDP } \\
\xi(k) \%(\mathrm{p}=0.5, \mathrm{n}=-0.1)\end{array}$ & 446105 & 488338.13 & 494343.81 & 510174.30 & 531096.92 & 555633.18 & 583182.25 & 613483.88 & 0.3398 \\
\hline Forecast GDP & 0 & -0.5529 & 0.3028 & 0.2188 & -0.2851 & & & & \\
$\xi(k) \%(\mathrm{p}=0.7, \mathrm{n}=0.0)$ & 446105 & 486507.81 & 501014.54 & 515953.83 & 531338.58 & 547182.07 & 563497.99 & 580300.41 & 0.6152 \\
\hline $\begin{array}{l}\text { Forecast GDP } \\
\xi(k) \%(\mathrm{p}=0.9, \mathrm{n}=0.0)\end{array}$ & 0 & -0.1760 & -1.0426 & -0.9116 & -0.3307 & & & & \\
\hline $\begin{array}{l}\text { Forecast GDP } \\
\text { by weighted average NGBM }\end{array}$ & 446105 & 489427.57 & 504107.80 & 519228.36 & 534802.45 & 550843.69 & 567366.08 & 584384.05 & 1.2451 \\
$\xi(k) \%$ & 0 & -0.7772 & -1.6664 & -1.5520 & -0.9848 & & & & \\
\hline
\end{tabular}

\section{CONCLUSION}

Overestimation or underestimation often occurs in forecasting practices. This study adopts weighted average $\operatorname{NGBM}(1,1)$ to balance the effect. Besides, a criterion proposed for assessing grey forecasting models includes in-sample and out-of-sample error. This study uses the weighted average $\operatorname{NGBM}(1,1)$ to forecast Taiwan's GDP. The results indicate that the proposed method performs better than any other $\operatorname{NGBM}(1,1)$ when in-sample and out-of-sample tests are taken into account. Besides, the forecast outcomes show that Taiwan's GDP is steadily growing. It may serve as valuable information for policy makers and investors.

\section{REFERENCES}

[1] K. L.Wen, Grey Systems: Modeling and Prediction, Arizona, Yang's, 2004.
[2] J. L. Deng, "Introduction of grey system," Journal of Grey System, vol.1, no. 1, pp.1-24, 1989.

[3] Z. X. Wang, "An optimized Nash nonlinear grey Bernoulli model for forecasting the main economic indices of high technology enterprises in China," Computers \& Industrial Engineering, vol. 64, pp. 780-787, 2013.

[4] H. Yong, "A new forecasting model for agricultural commodities," Journal of Agricultural Engineering Research, vol. 60, pp. 227-235, 1995.

[5] Q. Y. Xu and Y. H. Wen, "The application of Grey model on the forecast of passenger of international air transportation," Transportation Planning Journal, vol. 26, no. 3, pp. 525-555, 1997.

[6] S. Bahrami, R. A. Hooshmand, and M. Parastegari, "Short term electric load forecasting by wavelet transform and grey model improved by PSO algorithm," Energy, vol.72, pp. 434-42, 2014.

[7] S. C. Chang, H. C. Lai, and H. C. Yu, " A variable P value rolling Grey forecasting model for Taiwan semiconductor industry production," Technological Forecasting and Social Change, vol. 72, pp. 623-640, 2005.

[8] Z. Zhao, J. Z. Wang, J. Zhao, and Z. G. Su, "Using a grey model optimized by differential evolution algorithm to forecast the per capita annual net income of rural households in China," Omega, vol. 40, no 5. pp525-532, 2012. 
[9] H. Samet and A. Mojallal, "Enhancement of electric arc furnace reactive power compensation using Grey-Markov prediction method," in Proc. Iet Generation, Transmission and Distribution, vol. 8, pp. 626-36, 2014.

[10] Y. F. Wang, "Predicting stock price using fuzzy grey prediction system," Expert Systems with Applications, vol. 22, pp.33-39, 2002.

[11] A. W. L Yao and S. C. Chi, "Analysis and design of a taguchi-grey based electricity demand predictor for energy management systems," Energy Conversion and Management, vol. 45, no. 7, pp.1205-1217, 2004.

[12] M. Evans, "An alternative approach to estimating the parameters of a generalized grey verhulst model: An application to steel intensity of use in the UK," Expert Systems with Applications, vol. 4, pp. 1236-1244, 2014.

[13] C. I. Chen, " Application of the novel nonlinear grey bernoulli model for forecasting unemployment rate," Chaos, Solitons \& Fractals, vol. 37, no. 1, pp.278-287, 2008.

[14] C. I. Chen,, H. L. Chen, and S. P. Chen, "Forecasting of foreign exchange rates of Taiwan's major trading partners by novel nonlinea grey bernoulli model Ngbm $(1,1)$," Communications in Nonlinear Science and Numerical Simulation, vol. 13, no. 6, pp.1194-1204, 2008.

[15] C. I. Chen, P. H. Hsin, and C. S. Wu, "Forecasting Taiwan's major stock indices by the Nash nonlinear grey Bernoulli model," Experts Systems with Applications, vol. 37, pp.7557-7562, 2010.
[16] W. M. Ma, X. X. Zhu, and M. M. Wang, "Forecasting iron ore import and consumption of China using grey model optimized by particle swarm optimization algorithm," Resources Policy, vol. 38, pp. 613-620, 2013.

[17] L. Zhang, Y. Zheng, K. Wang, X. Zhang, and Y Zheng, "An optimized Nash nonlinear grey Bernoulli model based on particle swarm optimization and its application in prediction for the incidence of Hepatitis B in Xinjiang, China," Computers in Biology and Medicine, vol. 49, pp. 67-73, 2014.

[18] L. C. Hsu, "A genetic algorithm based nonlinear grey Bernoulli model for output forecasting in integrated circuit industry," Expert Systems with Applications, vol. 37, pp. 4318-4323, 2010.

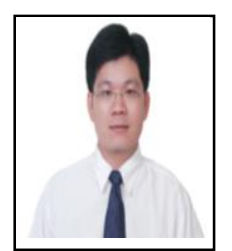

Pei-Han Hsin was born in Pingtung, Taiwan, R.O.C. in 1971. He received his B.Com. and M.B.A. degrees in international trade from National Chengchi University, Taipei, Taiwan, in 1985 and 1987, respectively. He received his $\mathrm{Ph} . \mathrm{D}$. degree in business management from National Sun Yat-Sen University, Kaohsiung, Taiwan, in 2010. From August 2000, he joined the Faculty of the Department of International Business at Cheng-Shiu University, Kaohsiung, Taiwan. From 2011, he has been an assistant professor in the Department of International Business at Cheng-Shiu University, Kaohsiung, Taiwan. His research interests include grey theory, grey forecasting, game theory and market micro-structure. 